\title{
TRATAMIENTO DE HIPERTROFIA MASETERINA UNILATERAL CON PLANO NEUROMIORELAJANTE Y APLICACIÓN DE TOXINA BOTULÍNICA TIPO A: CASO CLÍNICO
}

\section{Treatment of masseteric muscle hypertrophy with relaxing occlusal splint and botulinum toxin type a application: A case report}

\author{
Ordóñez-Pesántez María Gracia ${ }^{1 *}$, Barzallo-Sardi Vinicio Egidio ${ }^{2}$, Gómez-Aguilar Mishell Estephanía ${ }^{3}$. \\ ${ }^{1}$ Odontóloga, Residente en la Especialidad de Ortodoncia - Universidad Central del Ecuador. Miembro de la Sociedad \\ Ecuatoriana de Ortodoncia y Ortopedia \\ 2 Docente de la Facultad de Odontología - Universidad de Cuenca y Universidad de las Américas. Miembro de la Sociedad \\ Ecuatoriana de Ortodoncia y Ortopedia. \\ ${ }^{3}$ Odontóloga, Residente en la Especialidad de Rehabilitación Oral - Universidad Peruana Cayetano Heredia. \\ maria_2412m4@hotmail.com
}

\begin{abstract}
Resumen
La hipertrofia maseterina es una condición benigna en la que existe un aumento en el tamaño del músculo masetero, la misma que puede presentarse de forma uni o bilateral. No se le ha asignado etiología clara, aunque se presume que es debido a factores funcionales propios de cada individuo. El plano neuromiorelajante se considera una indicación terapéutica muy frecuente en múltiples patologías o trastornos de la unidad cráneocervicomandibular. Esta herramienta es la más utilizada para tratar trastornos témporomandibulares (TTM) y bruxismo. Así también, la toxina botulínica tipo A es una neurotoxina que en cantidades adecuadas es empleada de manera terapéutica o como producto cosmético por su capacidad de producir una parálisis muscular de manera temporal indicándose también en la hipertrofia maseterina. El uso de un plano de relajación y la aplicación de toxina botulínica han sido mayoritariamente estudiados por separados, pero el uso en conjunto podría suponer una mejora en los resultados como tratamiento en conjunto.

Una paciente de 30 años acude a la consulta por presentar hipertrofia maseterina unilateral derecha, el tratamiento de elección fue la instalación de un plano neuromiorelajante de uso 24 horas juntamente con la aplicación de toxina botulínica tipo A, después de un mes de la aplicación de esta se observó una mejoría evidente en la estética, sin embargo, a los cuatro meses de la aplicación cumpliendo este medicamento con su vida media se evidenció una recidiva importante.
\end{abstract}

Palabras clave: Bruxismo,férulas oclusales, hipertrofia, músculo masetero, toxina botulínica tipo A .

\begin{abstract}
Masseter hypertrophy is a benign condition which presents an enlargement of the masseter muscle that can occur unilaterally or bilaterally. Its etiology is unknown or idiopathic, although it is presumed to be caused by functional factors specific to everyone. The relaxing occlusal splint is considered a very frequent therapeutic indication in multiple pathologies or disorders of the cranio-cervicomandibular unit. This is the most used tool to treat temporomandibular disorders (TMD) and bruxism. Likewise, Botulinum Toxin Type A is a neurotoxin that in suitable amounts is used therapeutically or as a cosmetic product due to its ability to produce temporarily muscle paralysis, also being indicated in masseterine hypertrophy. The use of a relaxing occlusal splint and the application of botulinum toxin have been mostly studied separately, but the simultaneous use of these two therapeutic techniques could lead to an improvement in the results.

A 30 year old patient came into consult due to a unilateral masseteric hypertrophy of the right side, the treatment was the use of a relaxing occlusal splint during 24 hours complemented by injections of Botulinum Toxin type A, after a month of this application a noticeable improvement in facial aesthetics was observed, however after four months of the first application, a significant relapse was evident due to the drug's half-life.
\end{abstract}

Key words: Hypertrophy, masseter muscle, Botulinum toxins Type A, bruxism, occlusal splint. 


\section{Introducción}

La hipertrofia maseterina se define como el crecimiento excesivo de la masa muscular del músculo masetero, presenta una incidencia entre los 20 y 40 años sin predilección de sexo, su presentación es asintomática y de crecimiento lento; causa un aumento en tamaño del ángulo mandibular, provocando una morfología antiestética a este nivel y puede presentarse de forma uni o bilateral. Su etiología es de carácter desconocido o no está debidamente esclarecida, pero puede atribuirse a factores como el estrés, bruxismo, masticación con fuerzas excesivas y repetitivas, alteraciones oclusales y articulares también pueden causar desarmonías a nivel de los músculos de la región. ${ }^{1-7}$

Según la forma clínica Yun Xie y Cols. ${ }^{3}$ clasifican a la hipertrofia maseterina en cinco tipos de acuerdo con su estado de contracción muscular:

- Tipo I: Mínimo abultamiento sin evidencia clínica.

- Tipo II: Clínicamente evidenciable como un único abultamiento localizado.

- Tipo III: Clínicamente evidenciable como abultamiento doble mostrándose como dos haces separados longitudinales del mismo o distinto tamaño.

- Tipo IV: Clínicamente evidenciable como un abultamiento triple, como tres haces longitudinales.

- Tipo V: Clínicamente evidenciable como un solo abultamiento excesivo no delimitado o difuso.

El plano o férula oclusal es un aparato ortopédico intraoral removible elaborado a base de resina acrílica dura, muy utilizado para el tratamiento de trastornos témporomandibulares (TTM), que es instalado en los dientes de una de las arcadas para proporcionar un contacto simultáneo y homogéneo con sus respectivas piezas antagonistas, además que aumenta la dimensión vertical. Específicamente para el tratamiento del bruxismo se debe conseguir contactos únicamente en el sector anterior del plano, dejando libre de contactos en el sector posterior del mismo, debido a que se busca conseguir una relajación de los músculos masticatorios en movimientos céntricos y excéntricos, así mismo se ha demostrado que el aumento de la dimensión vertical puede disminuir la actividad muscular y los síntomas. ${ }^{8-11}$

La Toxina Botulínica tipo A (TBA) ha sido empleada para tratar distonías a nivel cervical y cada vez su uso es mayor para mejorar el contorno inferior del rostro y sintomatología dolorosa en casos de hipertrofia maseterina. Diferentes dosis son aplicadas en forma inyectable con técnicas apropiadas y seguras en esta región. 5,6,12,13

Da-Hye $\mathrm{Kim}^{4}$ menciona 6 zonas de subdivisión del músculo masetero (Fig. 1), siendo las zonas IV y V las más adecuadas para la aplicación de la toxina botulínica, las zonas I, II y III están asociadas a lesión del conducto parotídeo y la zona VI no está relacionada con la hipertrofia maseterina. Para la aplicación de la TBA Nam-Ho Kim ${ }^{5}$ describió una zona de seguridad (Fig. 2) que se delimita trazando una línea horizontal desde la comisura labial hacia el lóbulo de la oreja y otra línea paralela que pasa por el borde inferior de la mandíbula, dos líneas verticales paralelas que pasan por delante y por detrás del músculo masetero, la zona que queda entre estas líneas es la llamada zona de seguridad. ${ }^{5,6}$

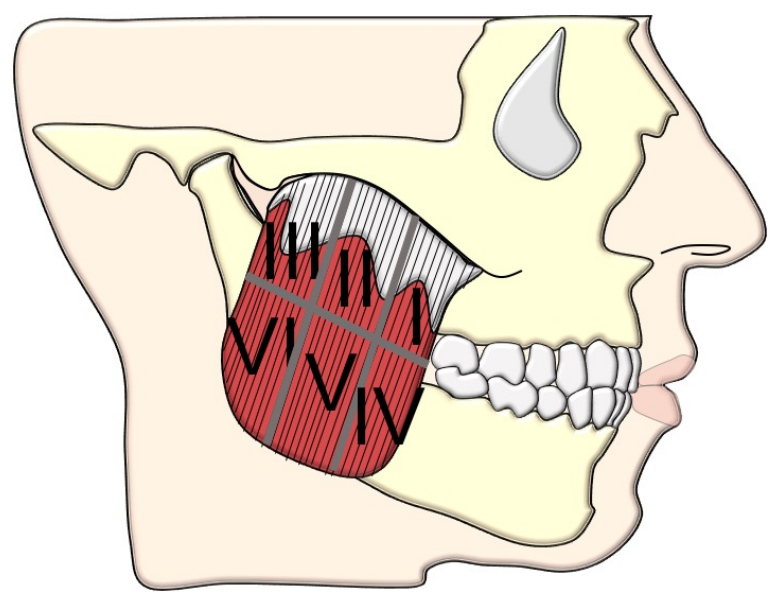

Fig. 1. Zonas de subdivisión del músculo masetero. Figura adaptada de Da-Hye Kim ${ }^{4}$

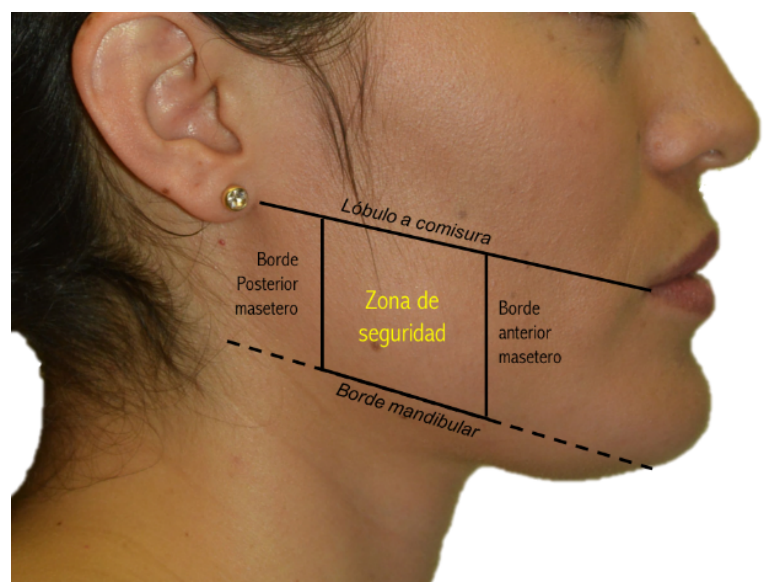

Fig. 2. Zona de seguridad para aplicación de TBA - Nam-Ho Kim ${ }^{5}$

El mecanismo de acción de la TBA es inhibir la acción de la acetilcolina, pues la TBA intercepta la unión de las proteínas necesarias para la liberación de esta, produciendo una denervación química que provoca una parálisis temporal al inhibir la contracción muscular, justificando así su empleo en este tipo de patologías. ${ }^{13}$ 


\section{Caso clínico}

Paciente de sexo femenino de 30 años, derivada por su cirujano máxilo-facial para tratamiento de hipertrofia maseterina unilateral. Después de la anamnesis, examen clínico y análisis radiográfico (panorámica, cefálica lateral y ecografía), se evidenció una marcada hipertrofia maseterina tipo II de lado derecho (Fig. 3A) y dolor hemifacial derecho 5 en la escala EVA, sin dolor articular. El tratamiento por parte del ortodoncista consistió en la instalación de un plano neuromiorelajante superior (Fig. 4A-B), controles de una vez por semana durante el primer mes, dos controles en el segundo mes y a partir del tercer mes controles mensuales durante 6 meses; simultáneamente se realizó la interconsulta con la dermatóloga, quien luego de un estudio conjunto efectuó la aplicación de 50 U de Toxina botulínica tipo A $\operatorname{Botox}(\mathrm{R})$ en la "zona de seguridad" del músculo masetero del lado derecho de la siguiente manera: $25 \mathrm{U}$ en la zona IV, $10 \mathrm{U}$ en la zona $\mathrm{V}$ descritas por Da - Hye $\mathrm{Kim}^{4}$ y $15 \mathrm{U}$ en dirección al borde inferior mandibular descrito por Nam - Ho $\mathrm{Kim}^{5}$ (Fig. 4). La aplicación se realizó en tres ocasiones con un margen de separación de 4 meses entre cada una y con el uso simultáneo del plano neuromiorelajante durante 24 horas al día, retirándose únicamente para el desayuno, almuerzo y cena.

\section{Discusión}

En casos de hipertrofia maseterina uni o bilateral, los pacientes acuden a la consulta en la gran mayoría porque su estética facial se ve comprometida, sin embargo, a este problema se le suman otros síntomas como es el dolor miofacial con relación a la región hipertrofiada, dolor articular e incluso dificultad para abrir la boca en algunos casos. A pesar de que no hay una etiología definida sobre la hipertrofia maseterina, se le atribuye a diferentes causas como la maloclusión, bruxismo y TTM. ${ }^{1,2,14,17}$

En el presente reporte de caso, los resultados obtenidos en la paciente mediante el uso simultáneo de plano neuromiorelajante y la aplicación de Toxina Botulínica Tipo A fueron favorables, debido a la disminución considerable de dolor y a que se obtuvo una mejoría en la estética facial en concordancia con el estudio de González y cols. ${ }^{1}$ quienes manifiestan que el uso inicial de PNMR y posterior aplicación de TBA permiten obtener una adecuada respuesta en el control de crecimiento de los músculos maseteros conservando armonía facial.

Al respecto Baş $\mathrm{B}$ y cols. ${ }^{18}$ indican que en los dos casos clínicos de hipertrofia maseterina unilateral con inyección de toxina botulínica tipo A los resultados fueron satisfactorios en cuanto a la estética. De igual manera el estudio de Castro y cols. ${ }^{14}$ reveló que todos los pacientes

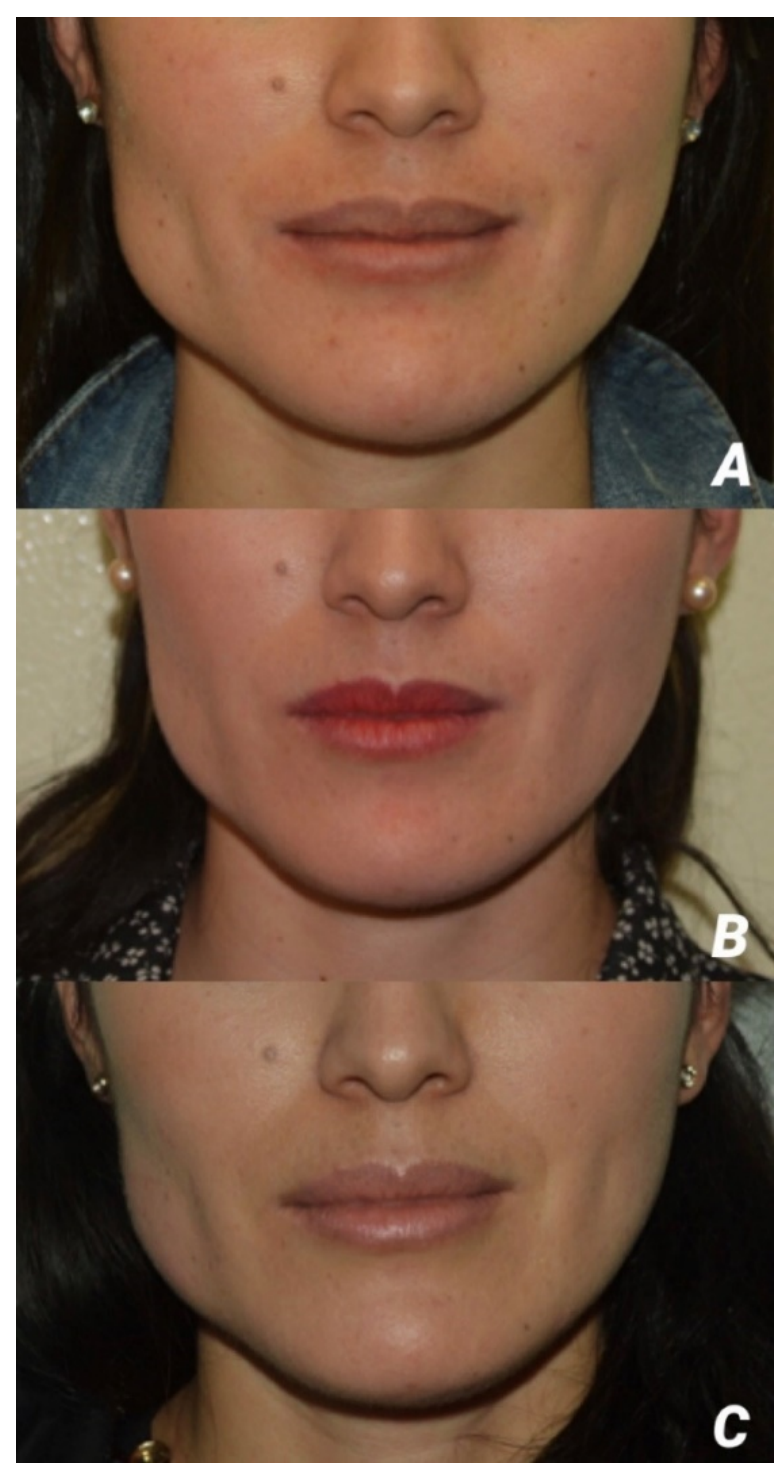

Fig. 3. Evolución de la paciente. A. Pre-tratamiento hipertrofia maseterina unilateral derecha. B. Post-tratamiento: 1 Mes posterior a la aplicación de TBA, disminución hipertrofia maseterina. C. PostTramiento: 4 meses posteriores a la primera aplicación de TBA, recidiva.

que fueron incluidos en el mismo muestran una disminución favorable de la hipertrofia maseterina, inclusive una mejora significativa en cuanto al dolor en los casos que lo reportaban.

Von Lindern y cols. ${ }^{19}$ afirman que existió mejora en cuanto al dolor orofacial en un $91 \%$ de los pacientes después de la aplicación de TBA. Por otro lado, Baker y cols. ${ }^{19}$ concluyen que la aplicación de TBA es un método seguro y efectivo con un gran beneficio para tratar el dolor orofacial, aunque tiene sus limitantes pues este es multifactorial, por lo que la inyección de TBA sería un tratamiento paliativo temporal del problema más no una solución definitiva. 

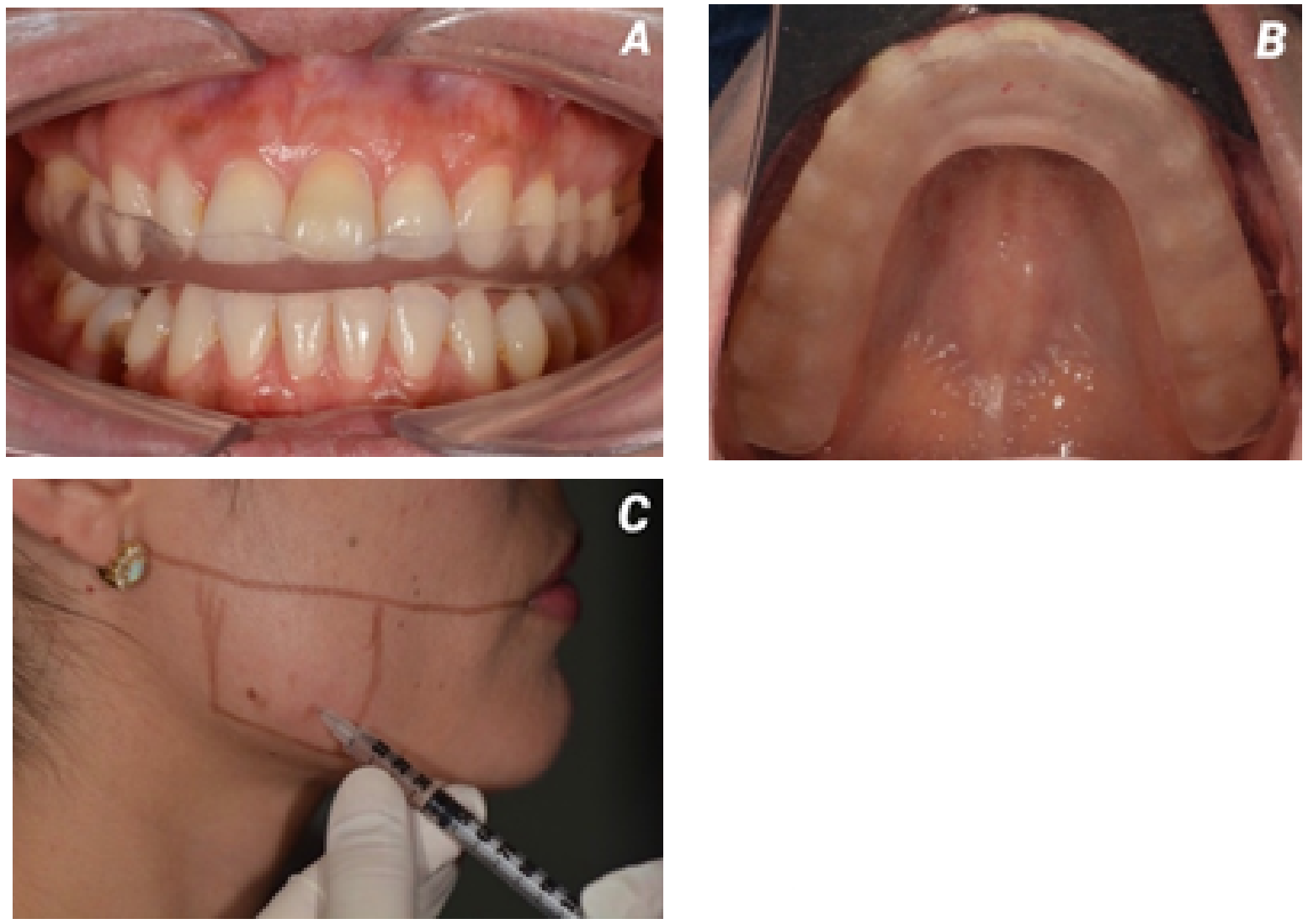

Fig. 4. Plano neuromiorelajante / Aplicación de TBA. A. Instalación de plano neuromiorelajante. B. Plano neuromiorelajante para bruxismo. C. Aplicación de toxina botulínica Tipo A en la "Zona de Seguridad".

Así mismo, Martínez-Pérez ${ }^{20}$ manifiesta que la aplicación de TBA es un tratamiento eficaz y seguro con algunas limitaciones, dentro de las cuales es la vida media de su efecto terapéutico lo mismo que podría hacer que el paciente requiera de este tipo de tratamiento múltiples veces que resultaría incomodo e incluso podría crear resistencias.

En cuanto al pronóstico de duración del efecto del tratamiento con TBA, Baş B y cols. ${ }^{18}$ mencionan que la vida media del medicamento es de 4 a 6 meses, provocando que el músculo regrese a su condición original después de ese tiempo, lo cual es coincidente con lo encontrado en nuestra paciente.

A pesar de no encontrarse reportes similares al presente en cuanto a la utilización de plano neuromiorelajante simultáneo a la aplicación de TBA, en este reporte de caso se utiliza la sinergia de estos dos elementos como tratamiento, no siendo una terapéutica definitiva, pero que resulta una excelente opción para tratar sintomatología, así como para obtener evidentes resultados estéticos para quienes buscan opciones menos invasivas.

\section{Conclusiones}

Los resultados terapéuticos del tratamiento de la hipertrofia maseterina con uso de plano neuromiorelajante conjuntamente a la aplicación de toxina botulínica tipo A implican una mejora considerable en la estética y disminución del dolor muscular. Sin embargo, el solo uso de TBA no podría establecerse como un tratamiento definitivo pues existe recidiva en un corto periodo de tiempo posterior a su aplicación.

Conflicto de interés: Los autores declaran no tener conflicto de interés.

\section{Referencias}

1 González M, Miranda L, Malagón H, González V. Uso de toxina botulínica para tratamiento de la hipertrofia del músculo masetero. Cir Plast Iberolatinoam. 2012;38(3): 297-302. 
2 Yanine N, Araya I, Cornejo M, Villanueva J. Tratamiento quirúrgico de hipertrofia maseterina: Reporte de un caso con 20 seguimiento de seis años. Rev Esp Cirug Oral y Maxilofac. 2009;31(6): 381-384.

3 Xie Y, Zhou J, Li H, Cheng C, Herrler T, Li Q. Classification 21 of Masseter Hypertrophy for Tailored Botulinum Toxin Type A Treatment. Plast Reconstr Surg. 2014;134(2):209e$218 \mathrm{e}$.

4 Kim DH, Hong HS, Won SY, et al. Intramuscular nerve distribution of the masseter muscle as a basis for botulinum toxin injection. J Craniofac Surg. 2010;21:588-591.

5 Kim NH, Chung JH, Park RH, Park JB. The use of botulinum toxin type $\mathrm{A}$ in aesthetic mandibular contouring. Plast Reconstr Surg. 2005;115:919-930.

6 Kim NH, Chung JH, Park RH, Park JB. Botulinum Toxin Type A for the Treatment of Hypertrophy of the Masseter Muscle. Plast. Rec. Surg. 2010; 125 (6): 1693.

7 Young K, Taek S. The Change of Maximum Bite Force after Botulinum ToxinType A Injection for Treating Masseteric Hypertrophy. Plast. Rec. Surg. 2007; 120 (6):1662.

8 Santander H, Santander M, Valenzuela S y cols. Después de cien años de uso: ¿las férulas oclusales tienen algún efecto terapéutico?. Rev Clin Periodoncia Implantol Rehabil Oral. 2011;4(1):29-35.

9 Okeson JP. Tratamiento de oclusión y afecciones temporomandibulares. Madrid: Elsevier; 2008.

10 Saavedra J, Balarezo J, Castillo D. Férulas Oclusales. Rev Estomatol Herediana. 2012;22(4): 242-246.

11 Espinar E, Alfonso MV, Chaqués J, Martín A, Solano E. Férulas oclusales como principio de obtención del diagnóstico en relación céntrica en ortodoncia. Manejo Clínico. Rev Esp Ortod. 2003;33: 41-49.

12 González M.C, Müller B. Estudio clínico de la Hipertrofia Maseterina - Revisión de la Literatura. Acta Odontológica Venezolana. 1998;36(2).

13 Lang A. History an uses of botox (Botulinum Toxin Type A). Lippincotts Case Manag. 2004;9: 109-112.

14 Castro W, Gomez R, da Silva Oliveira J, Moura M, Gomez $\mathrm{R}$. Botulinum toxin type A in the management of masseter muscle hypertrophy. J Oral Maxillofac Surg. 2005;63:20-4.

15 Tanaka E, Detamore M, Mercuri L. Degenerative disorders of the temporomandibular joint: etiology, diagnosis and treatment. J Dent Res. 2008;87(4): 296-307.

16 Moore A, Wood G. The medical management of masseteric hypertrophy with botulinum toxine type A. Br J Oral Maxillofac Surg. 1994;32: 26-28.

17 Rončevič, R. Masseter Muscle Hypertrophy Aetiology and Therapy. J Max-Fac Surg. 1986;14: 344-348.

18 Baş B, Özan B, Muğlalı M, Çelebi N. Treatment of maseteric hypertrophy with botulinum toxin: A report of two cases. Med Oral Patol Oral Cir Bucal. 2010;15(4): 649-652.

19 Von Lindern J, Niederhagen B, Bergé S, Appel T. Type A botulinum toxin in the treatment of chronic facial pain associated with masticatory hyperactivity. J Oral Maxillofac
Surg. 2003;61(7):774-78.

Baker J, Nolan P. Effectiveness of botulinum toxin type A for the treatment of chronic masticatory myofascial pain: A case series. JADA. 2016;148(1):33-39.

Martínez-Pérez D. Toxina botulínica y su empleo en la patología oral y maxilofacial. Rev Esp Cirug Oral y Maxilofac. 2004; 26(3):149-154.

Recibido: 03 de septiembre de 2020

Aceptado: 01 de diciembre de 2020 
